\title{
Epigenetically modified nucleotides in chronic heroin and cocaine treated mice
}

Mu-Rong Chao ${ }^{\mathrm{a}, \mathrm{b}}$, Domniki Fragou ${ }^{c^{*}}$, Panos Zanos $^{\mathrm{d}^{*}}$, Chiung-Wen Hu$^{\mathrm{e}}$, Alexis Bailey ${ }^{\mathrm{d}}$, Sofia Kouidou ${ }^{\mathrm{f}}$, Leda Kovatsi ${ }^{\mathrm{c}^{* *}}$

${ }^{a}$ Department of Occupational Safety and Health, Chung Shan Medical University, Taichung, 402, Taiwan

${ }^{b}$ Department of Occupational Medicine, Chung Shan Medical University Hospital, Taichung, 402, Taiwan

${ }^{c}$ Laboratory of Forensic Medicine and Toxicology, School of Medicine, Aristotle University of Thessaloniki, Thessaloniki 54124, Greece

${ }^{\mathrm{d}}$ Sleep, Chronobiology \& Addiction group, Faculty of Health and Medical Sciences, University of Surrey, Guildford, GU2 7XH, Surrey, UK.

${ }^{e}$ Department of Public Health, Chung Shan Medical University, Taichung, 402, Taiwan fLaboratory of Biological Chemistry, School of Medicine, Aristotle University of Thessaloniki, Thessaloniki 54124, Greece

*Equal contribution

\section{${ }^{* *}$ Corresponding author:}

Leda Kovatsi, Assistant Professor

Laboratory of Forensic Medicine \& Toxicology,

School of Medicine, Aristotle University of Thessaloniki, Greece

Tel: +30 2310 999222, e-mail: kovatsi@hotmail.com

\footnotetext{
Abbreviations: 5-caC, 5-carboxylcytosine; 5-fC, 5-formylcytosine; 5-hmC, 5-hydroxymethylcytosine; 5-hmdC, 5-methyl-2'deoxycytidine; 5-mC, 5-methylcytosine; 5-mdC, 5-methyl-2'-deoxycytidine; AP-1, activator protein 1 ; ${ }^{\circ} \mathrm{C}$, degrees centigrade; CpG, cytosine-phosphate-guanine; $d_{3}-5$-hmdC, $d_{3}-5$-hydroxymethyl-2'-deoxycytidine; $d_{3}-5$-mdC, $d_{3}-5$-methyl-2'-deoxycytidine; $\mathrm{dC}, 2^{\prime}$-deoxycytidine; ${ }^{15} \mathrm{~N}_{3}$-dC, ${ }^{15} \mathrm{~N}_{3}-2^{\prime}$-deoxycytidine DNA, deoxyribonucleic acid; HPLC, high performance liquid chromatography; i.p., intraperitoneal; LC-MS/MS, liquid chromatography/tandem mass spectrometry; MRM, multiple reaction monitoring; OPRM1, opioid receptor mu 1; PKC $\varepsilon$, protein kinase C epsilon; Sox10, Sex-determining region $Y$ - related high mobility group box 10; SP-1, specificity protein 1; TET1, ten-eleven translocation 1.
} 


\section{Abstract}

Epigenetic changes include the addition of a methyl group to the 5' carbon of the cytosine ring, known as DNA methylation, which results in the generation of the fifth DNA base, namely 5-methylcytosine. During active or passive demethylation, an intermediate modified base is formed, 5-hydroxymethylcytosine.

We have currenlty quantified 5-methylcytosine and 5-hydroxymethylcytosine in the liver and brain of mice treated with cocaine or heroin, using liquid chromatography/tandem mass spectrometry (LC-MS/MS). Our results show that global 5-methylcytosine levels are not affected by heroin or cocaine administration, neither in the liver nor in the brain. However, 5-hydroxymethylcytosine levels are reduced in the liver following cocaine administration, while they are not affected by cocaine in the brain or by heroin administration in the liver and brain.

Elucidation of the epigenetic phenomena that take place with respect to drug abuse and addiction, via quantitative analysis of different modified bases, may enable a better understanding of the underlying mechanisms and may lead to more personalized and effective treatment options.

Keywords: 5-hydroxymethylcytosine, 5-methylcytosine, cocaine, heroin, addiction, epigenetics

\section{Introduction}

The field of epigenetics has received increasing scientific interest during the last 15 years. Epigenetic modifications, such as DNA methylation and histone modifications (phosphorylation and acetylation), are heritable and alter the phenotype while the DNA sequence remains unaffected. These modifications result in changes in chromatin structure and DNA accessibility and therefore alter gene expression (Handy et al., 2011).

During DNA methylation, a methyl group is added to the carbon at the $5^{\prime}$ position of the cytosine ring forming the fifth DNA base, 5-methylcytosine (5-mC). The preferred sites for DNA methylation are CpG dinucleotide rich regions of the genome, called CpG islands. DNA methylation at gene promoter sites causes transcriptional silencing and affects the expression of genes that play a role in 
different biological processes, such as cell adherence, cell cycle, DNA repair, detoxification and apoptosis (Fragou et al., 2007, Richardson, 2003). Therefore, epigenetic phenomena are involved in various disease states and processes, including drug addiction (Schmidt et al., 2013, Hope et al., 2013, Fragou et al., 2013, Kovatsi et al., 2011, Fragou et al., 2011).

Histone modifications and DNA methylation of specific genes play a significant role in the underlying mechanisms of drug addiction and abuse (Maze et al., 2011, Sun et al., 2012, Nestler, 2014, Nielsen et al., 2012b, Shirazi et al., 2013). Methylation of the AP-1 (Activator protein 1) and SP1 (Specificity protein 1) binding sites and subsequent regulation of PKCe (Protein kinase $\mathrm{C}$ epsilon) expression and transcription is induced in the fetal rat heart by maternal exposure to cocaine (Meyer et al., 2009, Zhang et al., 2007, Zhang et al., 2009), while alterations in the global methylation status are observed in hippocampal neurons (Novikova et al., 2008). Furthermore, it has been recently found that cocaine represses protein phosphatase-1C $\beta$ in rat brain through DNA methylation. This process may affect memory and learning and may play a role in behavioural changes induced by the drug (Pol Bodetto et al., 2013). Hypermethylation of genes and alterations in gene expression in the brain is thought to play a crucial role in drug addiction processes (Nielsen et al., 2009, Carouge et al., 2010, Yoo et al., 2012, Gorelick et al., 2005). Even abstinence from cocaine self-administration has been shown to induce changes in the DNA methylation status of CpG islands in the promoter region of the Sox10 gene in the brain (Nielsen et al., 2012a). Furthermore, vulnerability to drug addiction and in particular heroin, has been associated with OPRM1 (Opioid receptor mu 1) gene expression (Nielsen et al., 2009). Hypermethylation of the promoter region of the gene in former heroin addicts reduced the expression of OPRM1 in lymphocytes, and this mechanism could explain a predisposal to heroin addiction vulnerability (Kovatsi et al., 2011, Nielsen et al., 2009, Yuferov et al., 2010, Bart et al., 2004). Since epigenetic phenomena are reversible, demethylating agents could be used as therapeutic tools against opioid addiction and this seems to be a promising field towards the development of new therapeutic strategies (Kovatsi et al., 2011, Fragou et al., 2011, Kouidou et al., 2010). 
In a recent study, the global DNA methylation levels in the liver and brain of mice dosed with cocaine and heroin were evaluated (Fragou et al., 2013). This study revealed tissue specific differences in the global methylation status between brain and liver. However, no difference in the overall content of \% 5-MedC was observed between dosed and control animals in either tissue (Fragou et al., 2013). This observation could be explained by the triggering of demethylation as a repair mechanism. Therefore, monitoring of the demethylation process could elucidate the underlying mechanism responsible for these findings and could provide a useful insight into the processes involved in drug addiction.

A modified base of increasing scientific interest is 5-hydroxymethylcytosine (5$\mathrm{hmC}$ ), which is considered an intermediate product in the process of DNA demethylation, either active or passive. In the active demethylation process, $5-\mathrm{mC}$ is oxidized to $5-\mathrm{hmC}$. $5-\mathrm{hmC}$ is subsequently converted to cytosine with the help of DNA methyltransferases, or is deaminated to 5-hydroxymethyluracil, which is then removed by base excision pathways. Another suggested pathway involves the further oxidation of 5-hmC to 5-formylcytosine (5-fC) by TET proteins and then to 5carboxylcytosine (5-caC). Removal of $5-\mathrm{caC}$ is achieved either by decarboxylation or base excision pathways. In the passive demethylation process, conversion of $5-\mathrm{mC}$ to 5-hmC does not allow maintenance of the methylation since DNA methyltransferase 1 , an enzyme responsible for propagation of methylation patterns, cannot copy 5hmC (Pfeifer et al., 2013).

$5-\mathrm{hmC}$ has only recently been studied in relation to various diseases, such as cancer and neurodegenerative disorders. A decrease in global 5-hmC levels has been reported in melanoma and liver, kidney, colon, brain, lung, prostate and breast cancer.

Global 5-hmC levels can be used as a prognostic, diagnostic or predictive biomarker. The global levels of $5-\mathrm{hmC}$ have been recently used to evaluate the effectiveness of anticancer agents (Laird et al., 2013). Furthermore, a global increase in 5-hmC levels in the cartilage of patients suffering from osteoarthritis, compared to normal tissue, was reported (Taylor et al., 2014). In a recent study on mice with fragile X-associated tremor/ataxia syndrome, it was shown that overall cerebellar 5$\mathrm{hmC}$ levels were increased compared to wild type animals and that the genomic 
regions with an altered $5-\mathrm{hmC}$ profile were the ones that are associated with transcription factors and genes that play an important role in neuronal function and development (Yao et al., 2014).

Research on epigenetics in drug abuse and addiction has mainly focused until today on the methylation status of specific genes, rather than global DNA methylation and therefore information on the global picture is limited (Tian et al., 2012). Furthermore, 5 -hmC, which is a modified base receiving increasing scientific interest, has only recently been studied in the context of drug abuse (Jayanthi et al., 2013). By elucidating the global epigenetic phenomena that underlie drug addiction or the adverse effects of drug abuse, we hope to be able to provide personalised therapeutic strategies, based on the selective reversal of these epigenetic alterations.

We have currently determined, by LC-MS/MS, two modified bases, namely 5$\mathrm{mdC}$ and 5 -hmdC, in the liver and brain of mice that received heroin, in a chronic 'intermittent' escalating dose paradigm and cocaine, in a chronic "binge" escalating dose paradigm. For the purpose of our study, we have used high-end methodology, capable of distinguishing 5-mdC from 5-hmdC (which have a similar chemical structure and cannot always be separated effectively) and of quantifying both at very low levels.

\section{Materials and Methods}

\subsection{Animals and drug administration paradigms}

The animals used and the chronic heroin/cocaine dosing scheme have been previously described in detail (Fragou et al., 2013).

Briefly, thirty six eight-week old, male C57BL/6J mice (B \& K Universal, Hull, UK), were used in the current study, which was conducted according to the protocols approved by the Home Office (Animals Act 1986) UK and the European Community Council Directive of the 24th Nov 1986 (86/609/EEC).

For the heroin experiment, eighteen animals were divided into two groups ( $n=9 /$ group) and received intraperitoneal (i.p.) injections of either saline or heroin for 7 days. Two intraperitoneal injections of saline or heroin were administered daily $(17.00$ and $09.00 \mathrm{~h})$, in accordance to previous opioid administration protocols 
(Bailey et al., 2010, Muller and Unterwald, 2004, Spanagel, 1995). The heroin-treated animals received $2 \times 1 \mathrm{mg} / \mathrm{kg} /$ injection on day $1,2 \times 2 \mathrm{mg} / \mathrm{kg} /$ injection on days 2 and $3,2 \times 4 \mathrm{mg} / \mathrm{kg} /$ injection on days 4 and 5 , and $2 \times 8 \mathrm{mg} / \mathrm{kg} /$ injection on days 6 and 7 . This chronic 'intermittent' escalating dose paradigm was used to mimic a common pattern of self-administration in human heroin abusers (Kreek et al., 2002). Animals were killed by decapitation following a 30-sec exposure to $\mathrm{CO}_{2}$ and tissues of interest, i.e., brain and liver, were removed and snap frozen in liquid nitrogen.

For the cocaine experiment, another eighteen $\mathrm{C} 57 \mathrm{BL} / 6 \mathrm{~J}$ mice, divided into two groups ( $n=9 /$ group) received intraperitoneal (i.p.) injections of either saline or cocaine for 14 days. An escalating dose 'binge' administration paradigm was used, as previously described by Bailey et al. (Bailey et al., 2005, Bailey et al., 2007, Bailey et al., 2008, Bailey et al., 2005, Schlussman et al., 2005), to mimic a common pattern of self-administration in human cocaine abusers (Tsukada et al., 1996). The cocainetreated animals received $3 \times 15 \mathrm{mg} / \mathrm{kg} /$ day on days $1-4,3 \times 20 \mathrm{mg} / \mathrm{kg} /$ day on days $5-8,3 \times 25 \mathrm{mg} / \mathrm{kg} /$ day on days 9-12, and $3 \times 30 \mathrm{mg} / \mathrm{kg} /$ day on days 13 and 14 . Animals were killed by decapitation following exposure to $\mathrm{CO}_{2}$ and tissues of interest (brain and liver) were dissected and immediately snap frozen in liquid nitrogen. Tissues were stored at $-80^{\circ} \mathrm{C}$ until use.

\subsection{DNA Extraction \& Hydrolysis}

Mice liver and brain DNA isolation was performed as previously described using the Qiagen DNA Buffer set and the Qiagen genomic tip (Fragou et al., 2013). Briefly, the tissues were homogenized in Buffer $G 2$ and then incubated with RNase A, RNase T1 and Proteinase K. The resulting homogenate was loaded onto the Qiagen genomic tip, which was previously equilibrated with Buffer QBT. The tip was washed with Buffer QC and the DNA was subsequently eluted with Buffer QF. Enzymatic hydrolysis of DNA was performed as described by Hu et al. (Hu et al., 2013), spiked with $d_{3}-5-m d C$ and $d_{3}-5-h m d C$ (Toronto Research Chemicals) as internal standards, followed by LC-MS/MS analysis.

\subsection{Analysis of 5-mdC and 5-hmdC using LC-MS/MS}


Concentrations of 5-mdC and 5-hmdC in DNA were measured using a recently validated LC-MS/MS method by Hu et al. (Hu et al., 2014, manuscript submitted for publication) with some modifications. LC-MS/MS analysis was performed using an Agilent 1100 series HPLC system interfaced with a API 3000 triple quadrupole mass spectrometer with electrospray ionization (ESI) source (Applied Biosystems). Briefly, $20 \mu \mathrm{L}$ of prepared DNA sample were initially cleaned up using an automatic system, which consisted of a switching valve (2-position microelectric actuator from Valco) and a C18 trap column ( $33 \times 2.1 \mathrm{~mm}$ i.d., $5 \mu \mathrm{m}$, ODS-3, Inertsil). The mobile phase consisted of: (A) 3\% (v/v) methanol $(\mathrm{MeOH}) / 1 \mathrm{mM}$ ammonium acetate $(\mathrm{AA})$ and $(\mathrm{B})$ $75 \%(\mathrm{v} / \mathrm{v}) \mathrm{MeOH} / 1 \mathrm{mM} \mathrm{AA}$, at a flow rate of $200 \mu \mathrm{L} / \mathrm{min}$. The gradient program started with $100 \%$ A held for $4.7 \mathrm{~min}, 100 \%$ A to reach $100 \%$ B at $4.8 \mathrm{~min}, 100 \%$ B held for $1.2 \mathrm{~min}, 100 \% \mathrm{~B}$ to reach $100 \% \mathrm{~A}$ at $6.1 \mathrm{~min}$ and held for $8.9 \mathrm{~min}$. After automatic sample cleanup, the sample was automatically transferred onto a C18 analytical column (150 × $2.1 \mathrm{~mm}$ i.d., $5 \mu \mathrm{m}$, ODS-3, Inertsil). The column was washed with a mobile phase of (C) $5 \%(\mathrm{v} / \mathrm{v}) \mathrm{MeOH} / 1 \mathrm{mM}$ AA and (D) $100 \%$ of $50 \%(\mathrm{v} / \mathrm{v})$ $\mathrm{MeOH} / 1 \mathrm{mM}$ AA. The gradient program started with $100 \% \mathrm{C}$ held for $6.0 \mathrm{~min}, 100 \%$ C to reach $100 \% \mathrm{D}$ at $11.0 \mathrm{~min}, 100 \% \mathrm{D}$ to reach $100 \% \mathrm{C}$ at $11.6 \mathrm{~min}$ and held for 3.4 $\min$. The total run time was $15 \mathrm{~min}$.

The samples were analyzed in the positive ion multiple reaction monitoring (MRM) mode, and the transitions of the precursors to the product ions were as follows: 5-mdC (m/z $242 \rightarrow 126$ and $242 \rightarrow 109), \mathrm{d}_{3}-5-\mathrm{mdC}(\mathrm{m} / \mathrm{z} 245 \rightarrow 129), 5-\mathrm{hmdC}$ $(\mathrm{m} / \mathrm{z} 258 \rightarrow 142$ and $258 \rightarrow 124)$ and $\mathrm{d}_{3}-5-\mathrm{hmdC}(\mathrm{m} / \mathrm{z} 261 \rightarrow 145)$. The dwell times per channel were set at $100 \mathrm{~ms}$ for both the analytes and the internal standards. The optimized ESI-MS/MS conditions were as follows: needle voltage, $5000 \mathrm{~V}$; nebulizer gas flow, 10; curtain gas flow, 10; turbo gas flow, 8 heated at $450{ }^{\circ} \mathrm{C}$; collisionassisted-dissociation gas flow, 4; declustering potential voltage, $15 \mathrm{~V}$ for $5-\mathrm{mdC} / \mathrm{d}_{3}-5-$ $\mathrm{mdC}$ and $20 \mathrm{~V}$ for $5-\mathrm{hmdC} / \mathrm{d}_{3}-5-\mathrm{hmdC}$; focusing potential voltage, $150 \mathrm{~V}$ for 5$\mathrm{mdC} / \mathrm{d}_{3}-5-\mathrm{mdC}$ and $130 \mathrm{~V}$ for $5-\mathrm{hmdC} / \mathrm{d}_{3}-5-\mathrm{hmdC}$; collision energy, $15 \mathrm{~V}(\mathrm{~m} / \mathrm{z}$ $242 \rightarrow 126)$ and $50 \mathrm{~V}(\mathrm{~m} / \mathrm{z} 242 \rightarrow 109)$ for $5-\mathrm{mdC}$, and $15 \mathrm{~V}$ for $\mathrm{d}_{3}-5-\mathrm{mdC}, 20 \mathrm{~V}(\mathrm{~m} / \mathrm{z}$ $258 \rightarrow 142)$ and $35 \mathrm{~V}(\mathrm{~m} / z 258 \rightarrow 124)$ for $5-\mathrm{hmdC}$, and $20 \mathrm{~V}$ for $\mathrm{d}_{3}-5-\mathrm{hmdC}$. Peak widths were set at 0.7 Th (full width at half-maximum) for both Q1 and Q3. The limits 
of detection were $0.012 \mathrm{ng} / \mathrm{mL}$ on column (1.0 fmol in a $20 \mu \mathrm{L}$ injection volume) and $0.2 \mathrm{ng} / \mathrm{mL}$ on column (16 fmol) for $5-\mathrm{mdC}$ and $5-\mathrm{hmdC}$, respectively.

\subsection{Statistical Analysis}

All statistical analyses were carried out using the SPSS software V. 18. The Mann-Whitney U-test was used for group comparison, with a confidence interval of $95 \%$. Values are expressed as mean \pm SD $(n=9)$.

\section{Results}

Fig. 1 shows a representative LC-MS/MS chromatogram for 5 -mdC and 5-hmdC and their stable isotope-labeled internal standards of a hydrolysate from saline treated mice brain DNA. Data obtained from the two different control groups showed that the 5-mdC levels ranged between $3.4 \%$ and $3.8 \%$ in both tissues, whereas the 5-hmdC levels were significantly lower, 0.2-0.6 \% (Tables 1 \& 2).

Our results showed that chronic, escalating dose, heroin administration did not affect 5-mdC or 5-hmdC levels in the brain and liver (Table 1).

Chronic, "binge", escalating dose cocaine administration did not affect 5-mdC levels, neither in the brain nor the liver (Table 2). Nevertheless, it induced a statistically significant decrease of 5 -hmdC levels in the liver $(p=0.000)$, while no effect on 5-hmdC levels was observed in the brain of cocaine-treated animals (Table 2).

When the two different tissues were compared, the 5-mdC levels were always higher in brain, compared to liver, not only in the two control groups (cocaine control group: brain 3.7\%, liver 3.6\%, $p=0.0050$ and heroin control group: brain $3.8 \%$, liver $3.4 \%, p=0.000$ ), but also in the drug-treated groups (cocaine-treated group: brain $3.7 \%$, liver $3.5 \%, p=0.000$ and heroin treated group: brain $3.9 \%$, liver $3.4 \%$, $p=0.000)$.

Similar findings were observed for 5 -hmdC. Brain was in every case the tissue where this modified base was more abundant (cocaine control group: brain $0.6 \%$, liver $0.2 \%, p=0.000$; heroin control group: brain $0.3 \%$, liver $0.2 \%, p=0.000$; cocainetreated group: brain $0.6 \%$, liver $0.1 \%, p=0.000$; heroin-treated group: brain $0.3 \%$, liver $0.2 \%, p=0.000)$. 


\section{Discussion}

The LC-MS/MS method used in this study is precise and reliable ( $\mathrm{Hu}$ et al., 2013). The stable isotope-labeled internal standard method enhances accuracy for the determination of the 5-mdC and 5-hmdC content. There is limited information on global DNA methylation and hydroxymethylation induced by drug abuse and addiction. Most of the studies published until today, focus on specific genes (Barros et al., 2013), with a special interest in the OPRM1 (Nielsen et al., 2009, Yuferov et al., 2010, Nielsen et al., 2010). In one case it was reported that the OPRM1 gene was significantly hypermethylated not only in blood, but also in sperm-derived DNA, suggesting that this epigenetic phenomenon may be the mechanism by which opioid abuse and dependence phenotypes are transferred to the next generation (Chorbov et al., 2011). In another study, it was shown that in the brain of chronic opiate addicts, enhanced methylation of the OPRM1 DNA hinders the $\mu$-opioid receptor upregulation. Furthermore, the researchers reported that this epigenetic mechanism is triggered via a nucleotide exchange which introduces a new methylation site concluding that genetic alterations may lead to epigenetic modifications with functional consequences (Oertel et al., 2012).

Although DNA methylation in drug addiction has received increasing scientific interest over the last years, $5-\mathrm{hmC}$ has only recently been studied in this context by a scientific group that focused on methamphetamine-induced alterations in DNA methylation and hydroxymethylation in the striatal glutamate receptor promoter sequences (Jayanthi et al., 2013). It was reported that methamphetamine causes a decrease in the methylation and hydroxymethylation of the GluA1 and GluA2 promoter sequences. The impact of these changes on receptor gene expression and, as a result, on behaviour is not clear, although it is unlikely that they are responsible for the downregulation of striatal glutamate receptors observed following chronic methamphetamine use (Jayanthi et al., 2013).

In the current study, we have determined global DNA methylation and hydroxymethylation in brain and liver of mice following chronic heroin and cocaine administration. We have focused on the global picture and not on specific genes and we have targeted both modified nucleotides. 
According to our findings, the administration of heroin did not affect 5-mdC or 5 -hmdC, in neither of the tissues examined. These findings are in agreement, as far as 5-mdC is concerned, with the findings of our previous study (Fragou et al., 2013), where brain and liver samples from cocaine- and heroin-treated mice were analyzed by HPLC-DAD. However, the HPLC-DAD method used in our previous study could not discriminate between 5-mdC and 5-hmdC which might co-elute together with other modified bases such as 5-formylcytosine and 5-carboxycytosine, therefore no data on 5-hmdC were available from that study.

The measurement of 5-mdC alone is not sufficient to determine changes in the methylation status, i.e. changes in methylation might by slipping unobserved because they might be compensated by protective mechanisms which convert 5$\mathrm{mdC}$ to $5-\mathrm{hmdC}$. Therefore, the simultaneous determination of 5-mdC and $5-\mathrm{hmdC}$ is important to determine a clear and accurate picture of the methylation state of a tissue.

In the current study, chronic heroin administration had no effect on the 5-mdC and 5-hmdC content of liver or brain, and therefore we can safely conclude that there was no change in the overall methylation status in these tissues following heroin administration.

5-mdC was not affected by cocaine in either tissue. The fact that chronic cocaine administration had no effect on 5-mdC levels in the liver and brain was anticipated based on the findings of our previous study, according to which the particular cocaine administration paradigm left the 5-mdC levels unaffected in both tissues studied.

On the other hand, we found that chronic cocaine administration decreased 5hmdC specifically in the liver but not the brain. Indeed, decreased 5-hmC has been associated with hepatocellular carcinomas and downregulation of the expression of TET1 proteins, with tumor size and poor survival and with alpha-fetoprotein levels (Liu et al., 2013). Moreover, Ivanov et al. (Ivanov et al., 2013) suggest that 5-hmC plays a role in liver function and development and that it might be responsible for inter-individual differences in drug metabolism and toxicity. Thus, based on the aforementioned evidence and given that chronic cocaine administration in the present study induced severe liver toxicity, we propose that the reduction in 5-hmdC 
levels in the liver could be the underlying mechanism for liver toxicity induced by chronic cocaine administration (Ivanov et al., 2013).

In our study, the livers of cocaine-treated animals exhibited regions of necrosis and intense eosinophilia (Fig. 2). Furthermore, their \% weight, relative to body weight, was significantly higher, when compared to the saline treated animals (6.12 $\pm 0.50 \%$ vs $4.27 \pm 0.26 \%, p<0.001$ ) (Fragou et al., 2013).

The higher 5-mdC and 5-hmdC content in brain, compared to liver, currently reported, is in agreement with previous findings (Khare et al., 2012, Unterberger et al., 2009, Song et al., 2011) and has been reported both in rats and mice (Shimabukuro et al., 2006, Kriaucionis and Heintz, 2009), as well as in humans (Fuke et al., 2004, Nestor et al., 2012). This difference in tissue 5-hmdC levels has been attributed to differences in 5-hmdC loss in passive demethylation pathways. Therefore, tissues with a relatively low proliferation rate (such as brain) don't lose as much as $5-\mathrm{hmC}$ and therefore, have a higher $5-\mathrm{hmC}$ content. Differences in 5-hmdC content are likely to affect gene transcription, altering the phenotype of a tissue. For example, breast cancer cell lines exhibit reduced levels of global 5-hmC, concomitant with extensive promoter hypermethylation which has been shown to affect gene expression (Nestor et al., 2012).

According to the same study, global 5-hmC levels do not correlate with global 5-mC levels and inter-tissue variation is much larger for global 5-hmC, than for global 5-mC. These findings suggest that although a gene may be transcribed at the same level in several tissues, great differences may be encountered in 5-hmC levels, depending on the tissue. Thus, quantitative analysis of 5-hmC is necessary (Nestor et al., 2012).

The two control groups of animals used in the current study exhibited similar 5-mC and 5-hmC levels in the two examined tissues, with the exception of 5-hmC levels in brain which were significantly higher in the control group used in the cocaine experiment. This deviation can be attributed to the fact that the control group of animals used in the cocaine experiment received 3 injections of saline per day, for 14 days, and was therefore subjected to more stress compared to the control group of animals used in the heroin experiment, that received 2 injections of saline per day, for 7 days. Studies have shown that the ten-eleven translocation (TET) 
proteins, which oxidize 5-mC to 5-hmC, are activated under stress conditions (Chia et al., 2011).

Epigenetic mechanisms in addiction constitute a field with increasing scientific interest (Fragou et al., 2013). It was only recently shown that different factors (such as s-adenosylmethionine treatment) may modulate the development of addiction by modifying cocaine-induced DNA methylation. These epigenetic mechanisms have been proposed to be involved in different addiction vulnerability of different individuals (Anier et al., 2013). The complete unraveling of these mechanisms, which are reversible, promises the development of new therapeutic strategies for craving and relapse. Research is currently underway towards this direction (Tian et al., 2012) and a very recent study showed that the responsiveness to disulfiram (a known modulator of global methylation) administration for cocaine dependence is influenced by the patient's genotype for the MTHFR gene, which is involved in methyl group supply. In this case, the authors implied that the patient's genotype is important in customizing the treatment for cocaine dependence, specifically for each individual and therefore in increasing the efficacy of the treatment (Spellicy et al., 2013).

In conclusion, we have currently quantified the global 5-mdC and 5-hmdC DNA content in the liver and brain of mice treated with cocaine or heroin, by LC-MS/MS. Our results showed that there is no effect on the global methylation and hydroxymethylation status in the liver and brain of heroin-treated animals, nor in the brain of cocaine-treated animals. However, a reduction in the 5-hmdC DNA content in the liver of cocaine-treated mice was observed, which might be, at least partly, involved in the underlying mechanism of liver toxicity induced by cocaine.

It is clear that the quantitative assessment both of $5-\mathrm{mdC}$, as well as $5-\mathrm{hmdC}$ provides a better understanding of the epigenetic mechanisms underlying various disease states, including drug abuse and addiction. This insight might prove extremely useful in cases where a more effective, personalized treatment is required, or in cases where the reversal of the epigenetic phenomena that take place could lead to prevention and/or cure.

\section{References}


Anier, K., Zharkovsky, A., Kalda, A., 2013. S-adenosylmethionine modifies cocaineinduced DNA methylation and increases locomotor sensitization in mice. Int. J. Neuropsychopharmacol. 16, 2053-2066.

Bailey, A., Gianotti, R., Ho, A., Kreek, M.J., 2007. Downregulation of kappa-opioid receptors in basolateral amygdala and septum of rats withdrawn for 14 days from an escalating dose "binge" cocaine administration paradigm. Synapse 61, 820-826.

Bailey, A., Gianotti, R., Ho, A., Kreek, M.J., 2005. Persistent upregulation of muopioid, but not adenosine, receptors in brains of long-term withdrawn escalating dose "binge" cocaine-treated rats. Synapse 57, 160-166.

Bailey, A., Metaxas, A., Al-Hasani, R., Keyworth, H.L., Forster, D.M., Kitchen, I., 2010. Mouse strain differences in locomotor, sensitisation and rewarding effect of heroin; association with alterations in MOP-r activation and dopamine transporter binding. Eur. J. Neurosci. 31, 742-753.

Bailey, A., Metaxas, A., Yoo, J.H., McGee, T., Kitchen, I., 2008. Decrease of D2 receptor binding but increase in D2-stimulated G-protein activation, dopamine transporter binding and behavioural sensitization in brains of mice treated with a chronic escalating dose 'binge' cocaine administration paradigm. Eur. J. Neurosci. 28, 759-770.

Bailey, A., Yuferov, V., Bendor, J., Schlussman, S.D., Zhou, Y., Ho, A., Kreek, M.J., 2005. Immediate withdrawal from chronic "binge" cocaine administration increases mu-opioid receptor mRNA levels in rat frontal cortex. Brain Res. Mol. Brain Res. 137, 258-262.

Barros, M., Dempster, E.L., Illott, N., Chabrawi, S., Maior, R.S., Tomaz, C., Silva, M.A., Huston, J.P., Mill, J., Muller, C.P., 2013. Decreased methylation of the NK3 receptor coding gene (TACR3) after cocaine-induced place preference in marmoset monkeys. Addict. Biol. 18, 452-454.

Bart, G., Heilig, M., LaForge, K.S., Pollak, L., Leal, S.M., Ott, J., Kreek, M.J., 2004. Substantial attributable risk related to a functional mu-opioid receptor gene polymorphism in association with heroin addiction in central Sweden. Mol. Psychiatry 9, 547-549.

Carouge, D., Host, L., Aunis, D., Zwiller, J., Anglard, P., 2010. CDKL5 is a brain MeCP2 target gene regulated by DNA methylation. Neurobiol. Dis. 38, 414-424.

Chia, N., Wang, L., Lu, X., Senut, M.C., Brenner, C., Ruden, D.M., 2011. Hypothesis: environmental regulation of 5-hydroxymethylcytosine by oxidative stress. Epigenetics. 6, 853-856.

Chorbov, V.M., Todorov, A.A., Lynskey, M.T., Cicero, T.J., 2011. Elevated levels of DNA methylation at the OPRM1 promoter in blood and sperm from male opioid addicts. J. Opioid Manag. 7, 258-264.

Fragou,A.,Kovatsi,L.,Kouidou,S., 2007. DNA methylation and specific sequence motifs: association with genetic instability in p53 in cancer, and other loci in degenerative disorders and aging. Arist Univ Med J 34, 41-48.

Fragou, D., Fragou, A., Kouidou, S., Njau, S., Kovatsi, L., 2011. Epigenetic mechanisms in metal toxicity. Toxicol. Mech. Methods 21, 343-352.

Fragou, D., Zanos, P., Kouidou, S., Njau, S., Kitchen, I., Bailey, A., Kovatsi, L., 2013. Effect of chronic heroin and cocaine administration on global DNA methylation in brain and liver. Toxicol. Lett. 218, 260-265. 
Fuke, C., Shimabukuro, M., Petronis, A., Sugimoto, J., Oda, T., Miura, K., Miyazaki, T., Ogura, C., Okazaki, Y., Jinno, Y., 2004. Age related changes in 5-methylcytosine content in human peripheral leukocytes and placentas: an HPLC-based study. Ann. Hum. Genet. 68, 196-204.

Gorelick, D.A., Kim, Y.K., Bencherif, B., Boyd, S.J., Nelson, R., Copersino, M., Endres, C.J., Dannals, R.F., Frost, J.J., 2005. Imaging brain mu-opioid receptors in abstinent cocaine users: time course and relation to cocaine craving. Biol. Psychiatry 57, 15731582.

Handy, D.E., Castro, R., Loscalzo, J., 2011. Epigenetic modifications: basic mechanisms and role in cardiovascular disease. Circulation 123, 2145-2156.

Hope, B.T., Skinner, M.K., Kenny, P.J., Akbarian, S., 2013. Exploring the epigenetics of cocaine resistance. Nat. Med. 19, 136-137.

Hu, C.W., Lee, H., Chen, J.L., Li, Y.J., Chao, M.R., 2013. Optimization of global DNA methylation measurement by LC-MS/MS and its application in lung cancer patients. Anal. Bioanal Chem. 405, 8859-8869.

Hu, C.W., Chen, J.L., Hsu, Y.W., Yen, C.C., Chao, M.R., 2014. Trace analysis of methylated and hydroxymethylated cytosines in DNA by isotope-dilution LC-MS/MS: first evidence of DNA methylation in Caenorhabditis elegans. Manuscript submitted for publication.

Ivanov, M., Kals, M., Kacevska, M., Barragan, I., Kasuga, K., Rane, A., Metspalu, A., Milani, L., Ingelman-Sundberg, M., 2013. Ontogeny, distribution and potential roles of 5-hydroxymethylcytosine in human liver function. Genome Biol. 14, R83.

Jayanthi, S., McCoy, M.T., Chen, B., Britt, J.P., Kourrich, S., Yau, H.J., Ladenheim, B., Krasnova, I.N., Bonci, A., Cadet, J.L., 2013. Methamphetamine Downregulates Striatal Glutamate Receptors via Diverse Epigenetic Mechanisms. Biol. Psychiatry .

Khare, T., Pai, S., Koncevicius, K., Pal, M., Kriukiene, E., Liutkeviciute, Z., Irimia, M., Jia, P., Ptak, C., Xia, M., Tice, R., Tochigi, M., Morera, S., Nazarians, A., Belsham, D., Wong, A.H., Blencowe, B.J., Wang, S.C., Kapranov, P., Kustra, R., Labrie, V., Klimasauskas, S., Petronis, A., 2012. 5-hmC in the brain is abundant in synaptic genes and shows differences at the exon-intron boundary. Nat. Struct. Mol. Biol. 19, 10371043.

Kouidou, S., Kovatsi, L., Ioannou, A., 2010. Seeking the 5th Base of DNA using chromatographic methods of analysis. Curr Org Chem 14, 2268-2281.

Kovatsi, L., Fragou, D., Samanidou, V., Njau, S., Kouidou, S., 2011. Drugs of abuse: epigenetic mechanisms in toxicity and addiction. Curr. Med. Chem. 18, 1765-1774.

Kreek, M.J., LaForge, K.S., Butelman, E., 2002. Pharmacotherapy of addictions. Nat. Rev. Drug Discov. 1, 710-726.

Kriaucionis, S., Heintz, N., 2009. The nuclear DNA base 5-hydroxymethylcytosine is present in Purkinje neurons and the brain. Science 324, 929-930.

Laird, A., Thomson, J.P., Harrison, D.J., Meehan, R.R., 2013. 5-Hydroxymethylcytosine Profiling as an Indicator of Cellular State. Epigenomics 5, 655-669.

Liu, C., Liu, L., Chen, X., Shen, J., Shan, J., Xu, Y., Yang, Z., Wu, L., Xia, F., Bie, P., Cui, Y., Bian, X.W., Qian, C., 2013. Decrease of 5-hydroxymethylcytosine is associated with progression of hepatocellular carcinoma through downregulation of TET1. PLoS One 8, e62828. 
Maze, I., Feng, J., Wilkinson, M.B., Sun, H., Shen, L., Nestler, E.J., 2011. Cocaine dynamically regulates heterochromatin and repetitive element unsilencing in nucleus accumbens. Proc. Natl. Acad. Sci. U. S. A. 108, 3035-3040.

Meyer, K., Zhang, H., Zhang, L., 2009. Direct effect of cocaine on epigenetic regulation of PKCepsilon gene repression in the fetal rat heart. J. Mol. Cell. Cardiol. 47, 504-511.

Muller, D.L., Unterwald, E.M., 2004. In vivo regulation of extracellular signalregulated protein kinase (ERK) and protein kinase $B(A k t)$ phosphorylation by acute and chronic morphine. J. Pharmacol. Exp. Ther. 310, 774-782.

Nestler, E.J., 2014. Epigenetic mechanisms of drug addiction. Neuropharmacology 76 Pt B, 259-268.

Nestor, C.E., Ottaviano, R., Reddington, J., Sproul, D., Reinhardt, D., Dunican, D., Katz, E., Dixon, J.M., Harrison, D.J., Meehan, R.R., 2012. Tissue type is a major modifier of the 5-hydroxymethylcytosine content of human genes. Genome Res. 22, 467-477.

Nielsen, D.A., Hamon, S., Yuferov, V., Jackson, C., Ho, A., Ott, J., Kreek, M.J., 2010. Ethnic diversity of DNA methylation in the OPRM1 promoter region in lymphocytes of heroin addicts. Hum. Genet. 127, 639-649.

Nielsen, D.A., Huang, W., Hamon, S.C., Maili, L., Witkin, B.M., Fox, R.G., Cunningham, K.A., Moeller, F.G., 2012a. Forced Abstinence from Cocaine Self-Administration is Associated with DNA Methylation Changes in Myelin Genes in the Corpus Callosum: a Preliminary Study. Front. Psychiatry. 3, 60.

Nielsen, D.A., Utrankar, A., Reyes, J.A., Simons, D.D., Kosten, T.R., 2012b. Epigenetics of drug abuse: predisposition or response. Pharmacogenomics 13, 1149-1160.

Nielsen, D.A., Yuferov, V., Hamon, S., Jackson, C., Ho, A., Ott, J., Kreek, M.J., 2009. Increased OPRM1 DNA methylation in lymphocytes of methadone-maintained former heroin addicts. Neuropsychopharmacology 34, 867-873.

Novikova, S.I., He, F., Bai, J., Cutrufello, N.J., Lidow, M.S., Undieh, A.S., 2008. Maternal cocaine administration in mice alters DNA methylation and gene expression in hippocampal neurons of neonatal and prepubertal offspring. PLoS One 3, e1919.

Oertel, B.G., Doehring, A., Roskam, B., Kettner, M., Hackmann, N., Ferreiros, N., Schmidt, P.H., Lotsch, J., 2012. Genetic-epigenetic interaction modulates mu-opioid receptor regulation. Hum. Mol. Genet. 21, 4751-4760.

Pfeifer, G.P., Kadam, S., Jin, S.G., 2013. 5-Hydroxymethylcytosine and its Potential Roles in Development and Cancer. Epigenetics Chromatin 6, 10-8935-6-10.

Pol Bodetto, S., Carouge, D., Fonteneau, M., Dietrich, J.B., Zwiller, J., Anglard, P., 2013. Cocaine represses protein phosphatase-1Cbeta through DNA methylation and Methyl-CpG Binding Protein-2 recruitment in adult rat brain. Neuropharmacology 73, 31-40.

Richardson, B., 2003. DNA methylation and autoimmune disease. Clin. Immunol. 109, 72-79.

Schlussman, S.D., Zhou, Y., Bailey, A., Ho, A., Kreek, M.J., 2005. Steady-dose and escalating-dose "binge" administration of cocaine alter expression of behavioral stereotypy and striatal preprodynorphin mRNA levels in rats. Brain Res. Bull. 67, 169175.

Schmidt, H.D., McGinty, J.F., West, A.E., Sadri-Vakili, G., 2013. Epigenetics and psychostimulant addiction. Cold Spring Harb Perspect. Med. 3, a012047. 
Shimabukuro, M., Jinno, Y., Fuke, C., Okazaki, Y., 2006. Haloperidol treatment induces tissue- and sex-specific changes in DNA methylation: a control study using rats. Behav. Brain Funct. 2, 37.

Shirazi, J., Shah, S., Sagar, D., Nonnemacher, M.R., Wigdahl, B., Khan, Z.K., Jain, P., 2013. Epigenetics, drugs of abuse, and the retroviral promoter. J. Neuroimmune Pharmacol. 8, 1181-1196.

Song, C.X., Szulwach, K.E., Fu, Y., Dai, Q., Yi, C., Li, X., Li, Y., Chen, C.H., Zhang, W., Jian, X., Wang, J., Zhang, L., Looney, T.J., Zhang, B., Godley, L.A., Hicks, L.M., Lahn, B.T., Jin, P., He, C., 2011. Selective chemical labeling reveals the genome-wide distribution of 5-hydroxymethylcytosine. Nat. Biotechnol. 29, 68-72.

Spanagel, R., 1995. Modulation of drug-induced sensitization processes by endogenous opioid systems. Behav. Brain Res. 70, 37-49.

Spellicy, C.J., Kosten, T.R., Hamon, S.C., Harding, M.J., Nielsen, D.A., 2013. The MTHFR C677T Variant is Associated with Responsiveness to Disulfiram Treatment for Cocaine Dependency. Front. Psychiatry. 3, 109.

Sun, H., Maze, I., Dietz, D.M., Scobie, K.N., Kennedy, P.J., Damez-Werno, D., Neve, R.L., Zachariou, V., Shen, L., Nestler, E.J., 2012. Morphine epigenomically regulates behavior through alterations in histone $\mathrm{H} 3$ lysine 9 dimethylation in the nucleus accumbens. J. Neurosci. 32, 17454-17464.

Taylor, S.E., Smeriglio, P., Dhulipala, L., Rath, M., Bhutani, N., 2014. A global increase in 5-hydroxymethylcytosine levels marks osteoarthritic chondrocytes. Arthritis Rheumatol. 66, 90-100.

Tian, W., Zhao, M., Li, M., Song, T., Zhang, M., Quan, L., Li, S., Sun, Z.S., 2012. Reversal of cocaine-conditioned place preference through methyl supplementation in mice: altering global DNA methylation in the prefrontal cortex. PLoS One 7, e33435.

Tsukada, H., Kreuter, J., Maggos, C.E., Unterwald, E.M., Kakiuchi, T., Nishiyama, S., Futatsubashi, M., Kreek, M.J., 1996. Effects of binge pattern cocaine administration on dopamine D1 and D2 receptors in the rat brain: an in vivo study using positron emission tomography. J. Neurosci. 16, 7670-7677.

Unterberger, A., Szyf, M., Nathanielsz, P.W., Cox, L.A., 2009. Organ and gestational age effects of maternal nutrient restriction on global methylation in fetal baboons. J. Med. Primatol. 38, 219-227.

Yao, B., Lin, L., Street, R.C., Zalewski, Z.A., Galloway, J.N., Wu, H., Nelson, D.L., Jin, P., 2014. Genome-wide alteration of 5-hydroxymethylcytosine in a mouse model of fragile X-associated tremor/ataxia syndrome. Hum. Mol. Genet. 23, 1095-1107.

Yoo, J.H., Kitchen, I., Bailey, A., 2012. The endogenous opioid system in cocaine addiction: what lessons have opioid peptide and receptor knockout mice taught us? Br. J. Pharmacol. 166, 1993-2014.

Yuferov, V., Levran, O., Proudnikov, D., Nielsen, D.A., Kreek, M.J., 2010. Search for genetic markers and functional variants involved in the development of opiate and cocaine addiction and treatment. Ann. N. Y. Acad. Sci. 1187, 184-207.

Zhang, H., Darwanto, A., Linkhart, T.A., Sowers, L.C., Zhang, L., 2007. Maternal cocaine administration causes an epigenetic modification of protein kinase Cepsilon gene expression in fetal rat heart. Mol. Pharmacol. 71, 1319-1328. 
Zhang, H., Meyer, K.D., Zhang, L., 2009. Fetal exposure to cocaine causes programming of Prkce gene repression in the left ventricle of adult rat offspring. Biol. Reprod. 80, 440-448. 


\section{Figure legends}

Fig. 1. Chromatograms of 5-mdC and 5-hmdC in mouse brain DNA, as measured by LC-MS/MS. Multiple reaction monitoring transitions of $m / z \quad 242 \rightarrow 126$ (A) and $m / z$ $242 \rightarrow 109$ (B) for $5-m d C, m / z 245 \rightarrow 129$ (C) for $d_{3}-5-m d C, m / z 258 \rightarrow 142$ (D) and $m / z$ $258 \rightarrow 124$ (E) for 5 -hmdC, and $m / z \quad 261 \rightarrow 145$ (F) for $d_{3}-5-h m d C$. cps, counts per second.

Fig. 2. Hepatic necrosis in the liver of a male C57BL/6 mouse administered an escalating dose of cocaine (viewed under a X10 objective).

$\mathrm{CV}=$ Central Vein, $\mathrm{E}=$ Eosinophilic Cells, $\mathrm{G}=$ Ghost Cells, I=Inflammatory Infiltration 
Table 1: 5-mdC and 5-hmdC levels in the liver and brain of heroin treated and control mice.

\begin{tabular}{|c|c|c|c|c|c|c|c|c|}
\hline & \multicolumn{8}{|c|}{ Heroin } \\
\hline & \multicolumn{2}{|c|}{ 5-mdC Liver } & \multicolumn{2}{|c|}{ 5-mdC Brain } & \multicolumn{2}{|c|}{ 5-hmdC Liver } & \multicolumn{2}{|c|}{ 5-hmdC Brain } \\
\hline & Control & Treated & Control & Treated & Control & Treated & Control & Treated \\
\hline Mean (\%) & 3.4 & 3.4 & 3.8 & 3.9 & 0.2 & 0.2 & 0.3 & 0.3 \\
\hline St. Dev. & 0.1 & 0.1 & 0.2 & 0.2 & 0.01 & 0.01 & 0.03 & 0.03 \\
\hline CV (\%) & 3.2 & 2.9 & 4.5 & 4.9 & 6.7 & 8.9 & 10.6 & 10.5 \\
\hline$p$ value $(95 \%)$ & \multicolumn{2}{|c|}{0.730} & \multicolumn{2}{|c|}{0.489} & \multicolumn{2}{|c|}{0.863} & \multicolumn{2}{|c|}{0.863} \\
\hline
\end{tabular}


Table 2: $5-\mathrm{mdC}$ and 5-hmdC levels in the liver and brain of cocaine treated and control mice.

\begin{tabular}{|c|c|c|c|c|c|c|c|c|}
\hline & \multicolumn{8}{|c|}{ Cocaine } \\
\hline & \multicolumn{2}{|c|}{ 5-mdC Liver } & \multicolumn{2}{|c|}{ 5-mdC Brain } & \multicolumn{2}{|c|}{ 5-hmdC Liver } & \multicolumn{2}{|c|}{ 5-hmdC Brain } \\
\hline & Control & Treated & Control & Treated & Control & Treated & Control & Treated \\
\hline Mean (\%) & 3.6 & 3.5 & 3.7 & 3.7 & 0.2 & 0.1 & 0.6 & 0.6 \\
\hline St. Dev. & 0.1 & 0.1 & 0.1 & 0.1 & 0.01 & 0.004 & 0.02 & 0.03 \\
\hline CV (\%) & 3.9 & 2.6 & 2.4 & 2.3 & 5.6 & 6.4 & 2.8 & 5.6 \\
\hline$p$ value $(95 \%)$ & \multicolumn{2}{|c|}{0.161} & \multicolumn{2}{|c|}{0.931} & \multicolumn{2}{|c|}{0.000} & \multicolumn{2}{|c|}{0.489} \\
\hline
\end{tabular}



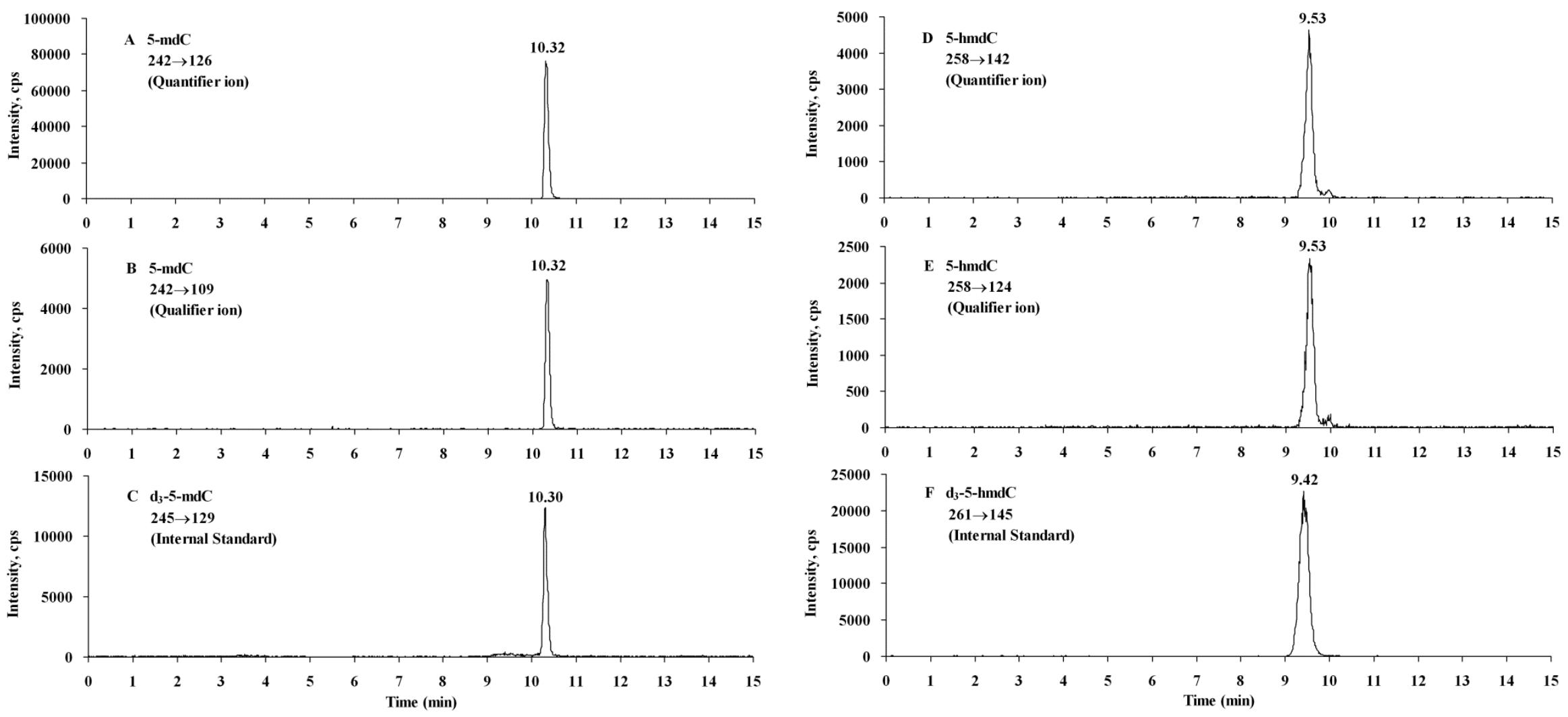

Fig. 1 


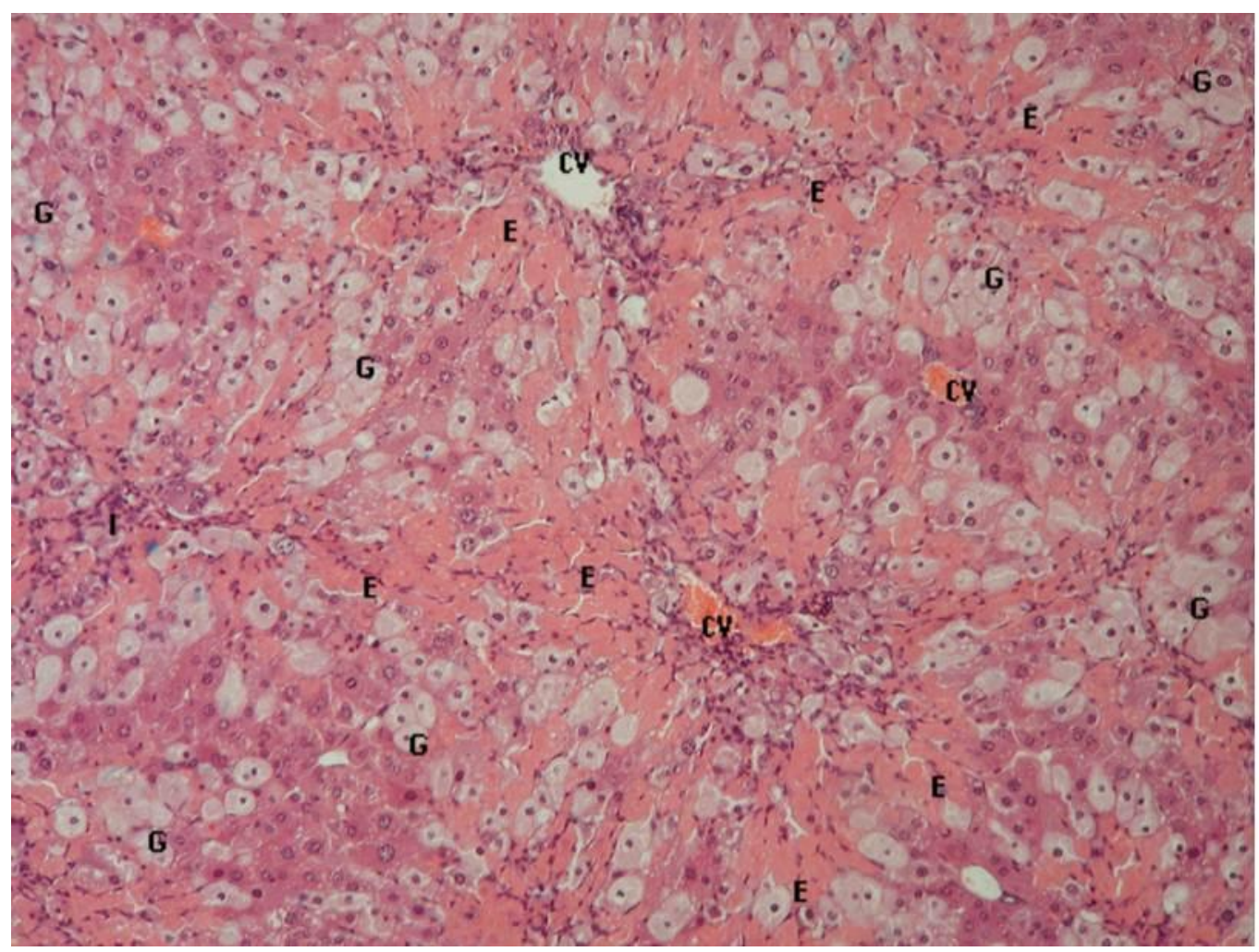

Fig. 2. Hepatic necrosis in the liver of a male $\mathrm{C} 57 \mathrm{BL} / 6$ mouse administered an escalating dose of cocaine (viewed under a 10 objective). CV, central vein, $E$, eosinophilic cells, G, ghost cells, I, inflammatory infiltration

Fig. 2 